\title{
Experimental and Numerical Study of a Printed Circuit Heat Exchanger
}

Minghui Chen ${ }^{\mathrm{a}}$, Shanbin Shi ${ }^{\mathrm{a}}$, Xiaodong Sun ${ }^{\mathrm{a}}$, Richard N. Christensen ${ }^{\mathrm{a}, 1}$, Isaac Skavdahl ${ }^{\mathrm{b}}$, Vivek Utgikar $^{\mathrm{b}}$, Piyush Sabharwall ${ }^{\mathrm{c}}$

${ }^{a}$ Nuclear Engineering Program, Department of Mechanical and Aerospace Engineering, The Ohio State University, Columbus, OH 43210, USA

${ }^{\mathrm{b}}$ Department of Chemical and Materials Engineering, University of Idaho, Moscow, ID 83844, USA

${ }^{\mathrm{c}}$ Idaho National Laboratory, Idaho Falls, ID 83415, USA

${ }^{1}$ Current address: Nuclear Engineering Program, University of Idaho, Idaho Falls, ID 83401, USA

\begin{abstract}
Printed circuit heat exchangers (PCHEs) are promising to be employed in very-high-temperature gascooled reactors (VHTRs) due to their high robustness for high-temperature, high-pressure applications and high compactness. PCHEs typically serve as intermediate heat exchangers (IHXs) that isolate the secondary loop from the reactor's primary system and hence must be sufficiently robust to maintain the system integrity during normal and off-normal conditions. In addition, the performance of the PCHE-type IHX could considerably affect the nuclear power plant overall operation since any transients on the secondary side would be propagated back to the reactor's primary coolant system via the IHX. It is therefore imperative to understand how the PCHE would dynamically respond to a variety of transients. In the current study, experiments were first conducted to examine the steady-state thermal performance of a reduced-scale straight-channel PCHE. A dynamic model benchmarked in a previous study was then used to predict the steady-state and transient behavior of the PCHE. The steady-state temperature profiles of the working fluids on both the hot and cold sides and in the solid plates of the heat exchanger were obtained, which served as the initial conditions for the transient simulations. The detailed dynamic
\end{abstract}


response of the straight-channel PCHE, subject to inlet temperature variations, helium mass flow variations, and combinations of both, was simulated and analyzed. In addition, two sets of transient tests, one for helium inlet temperature increase and the other for helium inlet temperature decrease, were experimentally carried out to assess the applicability of the dynamic model. Comparisons of the numerical results with the experimental data show that the dynamic model is successful in predicting the experimental transient scenarios. Although difference was observed between the numerical results and experimental data, it is suggested that the numerical solutions are sufficiently accurate and conservative and that the applicability of the dynamic model proposed for predicting the steady-state and transient performance of the straight-channel PCHE has been confirmed. Furthermore, both the numerical and experimental studies provide insights into the dynamic performance of the PCHE.

\section{Introduction}

Intermediate heat exchangers (IHXs) are widely proposed for use in the Generation IV nuclear systems, such as the very-high-temperature gas-cooled reactors (VHTRs). IHXs are required to transfer efficiently the thermal energy from the primary loop to a secondary fluid, which can be either helium, water/steam, or supercritical carbon dioxide (s- $\mathrm{CO}_{2}$ ). A printed circuit heat exchanger (PCHE) is one of the most promising IHX candidates for VHTRs due to its high robustness for high-temperature, high-pressure applications and high compactness [1, 2]. A number of studies have been conducted on PCHEs over the past decade. Nikitin et al. [3] experimentally investigated the heat transfer and pressure drop characteristics of a 3-kW thermal duty PCHE with a compactness of $1050 \mathrm{~m}^{2} / \mathrm{m}^{3}$ in an s-CO loop. The tested PCHE block consists of 12 hot plates and six cold plates with a unique double-banking configuration. Correlations were proposed to predict the local heat transfer coefficient and friction factor of the PCHE. Sabharwall et al. [4] evaluated a variety of heat exchanger types, including PCHE for applications in the Advanced High-Temperature Reactor (AHTR), a large fluoride salt-cooled hightemperature reactor (FHR) design. The authors summarized the potential issues related to heat exchanger 
materials, fabrication methods, system configuration, coolant properties and corrosion, and presented a conceptual design along with discussions of challenges associated with integrating a steam power cycle with the high-temperature reactor. Mylavarapu et al. [5] designed and fabricated two similar straightchannel PCHEs with a counter-current flow configuration using Alloy 617 plates for the PCHE core and Alloy $800 \mathrm{H}$ pipes for the headers. The thermal hydraulic performance of the PCHEs was tested in a hightemperature helium test facility (HTHF) at The Ohio State University. Both the Fanning friction factor and Nusselt number were obtained and comparisons between the experimental data and the results from available models were also carried out. It was found that the onset of laminar-to-turbulent flow transition regime seemed to occur earlier than that in a circular straight pipe. However, no heat transfer correlation was developed in their study.

Understanding PCHEs' dynamic performance is of significance in the design of a PCHE-type IHX for nuclear systems since the dynamic behavior of the PCHE and its coupling between the primary system and secondary loop are essential to the reactor operational safety. Therefore, a complete analysis of the transient response of a PCHE subject to multiple variations is imperative. Limited research on transient analyses of PCHEs, both numerical and experimental, has been conducted. Pra et al. [6] conducted experiments on a PCHE mock up to investigate its thermomechanical behavior. However, no dynamic model was developed and transients in temperature were not analyzed in their study. Ravindran et al. [7] designed a straight-channel PCHE for the Next Generation Nuclear Plant (NGNP) and conducted a loss of coolant accident (LOCA) simulation for the plant using RELAP5-3D. To simplify the complexity, the semicircular channels of the PCHE were mapped into rectangular geometries, which would lead to some distortions even in steady-state calculations. Although the literature on the PCHE transient analysis is sparse, a number of dynamic studies have been performed on plate-type heat exchangers for both steadystate and transient conditions. Since PCHEs are plate-type heat exchangers, the dynamic models developed for plate-type heat exchangers could be applied to PCHEs. Lakshmanan and Potter [8] developed a numerical model, named as “cinematic" model, based on energy balance by assuming there 
was no heat capacitance in solid plates and the fluid flow was plug flow. The "cinematic" model offers a convenient and fast method to simulate the steady-state and transient behaviors of plate-type heat exchangers. A general dynamic model that could be applied to plate-type heat exchangers with different geometrical dimensions was proposed by Sharifi et al. [9] based on the "cinematic” model. Experiments were conducted to assess the applicability of the dynamic model and it was indicated that numerical solutions and experimental data were in good agreement. Chen et al. [10] analyzed the dynamic response of an offset strip-fin plate-type heat exchanger used as a secondary heat exchanger (SHX) in a fluoridesalt-cooled high-temperature reactor (FHR). The adopted model was benchmarked by using a commercial software "DYNSIM" while no experimental data were available to validate the dynamic model. In a later

study, Chen et al. [11] performed both experiments and numerical simulations for a zigzag-channel PCHE utilizing the same numerical model. The dynamic model is successful in predicting the steady-state and transient behaviors of the zigzag-channel PCHE. Nonetheless, no transient simulation or experiment was carried out for the straight-channel PCHE.

In the current study, the dynamic model adopted from Sharifi et al. [9] and benchmarked by Chen et al. [10] was numerically used to predict the steady-state and transient behaviors of the reduced-scale straightchannel PCHE. The simulated transients, including the fluid inlet temperature variations and helium mass flow rate changes, were analyzed. Experiments were performed to assess the applicability of the dynamic model for simulating the performance of the straight-channel PCHE under helium-helium conditions.

\section{Printed Circuit Heat Exchanger and High-temperature Helium Test Facility}

\subsection{Printed Circuit Heat Exchanger}

A reduced-scale straight-channel PCHE was fabricated using 1.6-mm thick Alloy 617 plates for the heat exchanger core and Alloy $800 \mathrm{H}$ pipes for the headers. A total of 10 hot plates and 10 cold plates were 
diffusion bonded together to form a metal block with 12 straight channels in each plate [12]. Figure 1 shows one hot-side plate and one cold-side plate and Table I lists the basic geometric parameters of the fabricated PCHE. The shape of the straight flow channel is approximately semicircular with a diameter of approximately $2 \mathrm{~mm}$ and a pitch of $2.54 \mathrm{~mm}$ in the span-wise direction. The PCHE was designed in such a way that each side could withstand a maximum pressure of $3 \mathrm{MPa}$ in the HTHF. The flow passages in each plate of the PCHE were made by applying a photochemical etching technique, which used strong chemical etchants to remove the selected area on the surface of the plates. The actual PCHE assembly with four headers is shown in Fig. 2. The dimensions of the PCHE block are $305 \mathrm{~mm}$ in length by 102 $\mathrm{mm}$ in width by $73 \mathrm{~mm}$ in height.

Table I. Summary of the reduced-scale PCHE parameters [13]

\begin{tabular}{|l|c|c|}
\hline Parameter & Hot side & Cold side \\
\hline Channel pitch, mm & 2.54 & 2.54 \\
\hline Channel width, mm & 2.0 & 2.0 \\
\hline Plate thickness, mm & 1.63 & 1.63 \\
\hline Channel travel length, mm & 305 & 272 \\
\hline Number of plates & 10 & 10 \\
\hline Number of channels in each plate & 12 & 12 \\
\hline
\end{tabular}



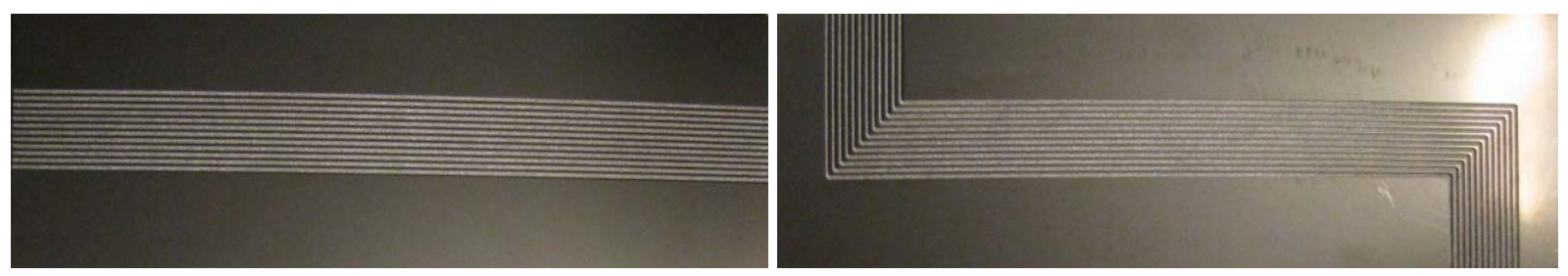

Fig. 1. One cold-side plate (left) and one hot-side plate (right) [13].

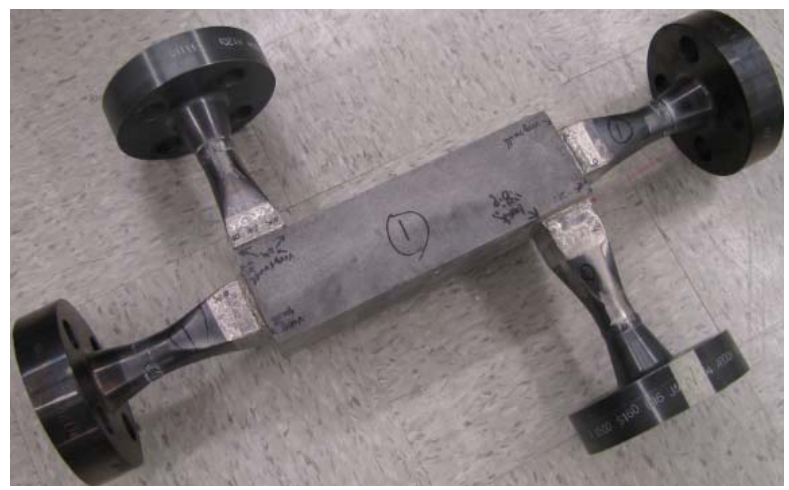

Fig. 2. PCHE with four headers assembled.

\subsection{High-temperature Helium Test Facility}

Experiments were performed to examine both the steady-state and transient performances of the straightchannel PCHE using the HTHF. Figure 3 shows a 3-D layout of the high-temperature helium test facility. The HTHF was constructed to facilitate performance testing of compact heat exchangers at temperatures and pressures up to $800^{\circ} \mathrm{C}$ and $3 \mathrm{MPa}$, respectively. Detailed description of the test facility was provided in Ref. [5]. Two straight-channel PCHEs, fabricated from Alloy 617 plates and Alloy $800 \mathrm{H}$ pipes, were installed in the HTHF. Two similar electric heaters, namely, pre-heater and main-heater, were also installed in the loop to heat helium to high temperatures during the elevated-temperature testing. Three heating elements were embedded on the inner surface of the ceramic fiber insulation of each of the heater, each element having a maximum capacity of approximate $7.6 \mathrm{~kW}$. Therefore, each heater has a maximum heating capacity of about $23 \mathrm{~kW}$. In addition, the maximum allowable temperature of the heating elements is $1,300{ }^{\circ} \mathrm{C}$. The helium flow in the test facility is driven by a single-stage double-acting air- 
drive booster, which has periodical fluctuations due to the reciprocating action of the booster. In addition to a helium surge tank, an inline air-drive pressure reducing valve was installed at a downstream location of the booster to damp the flow oscillations, as shown in Fig. 3. High-temperature helium is cooled by a helically-coiled cooler with chilled water as the coolant. The HTHF consists of a variety of instruments, high-temperature valves, flanges, and piping. To assure the measurement accuracy of the experimental data, all the measurement sensors and test equipment were calibrated against standards traceable to the National Institute of Standards and Technology (NIST).
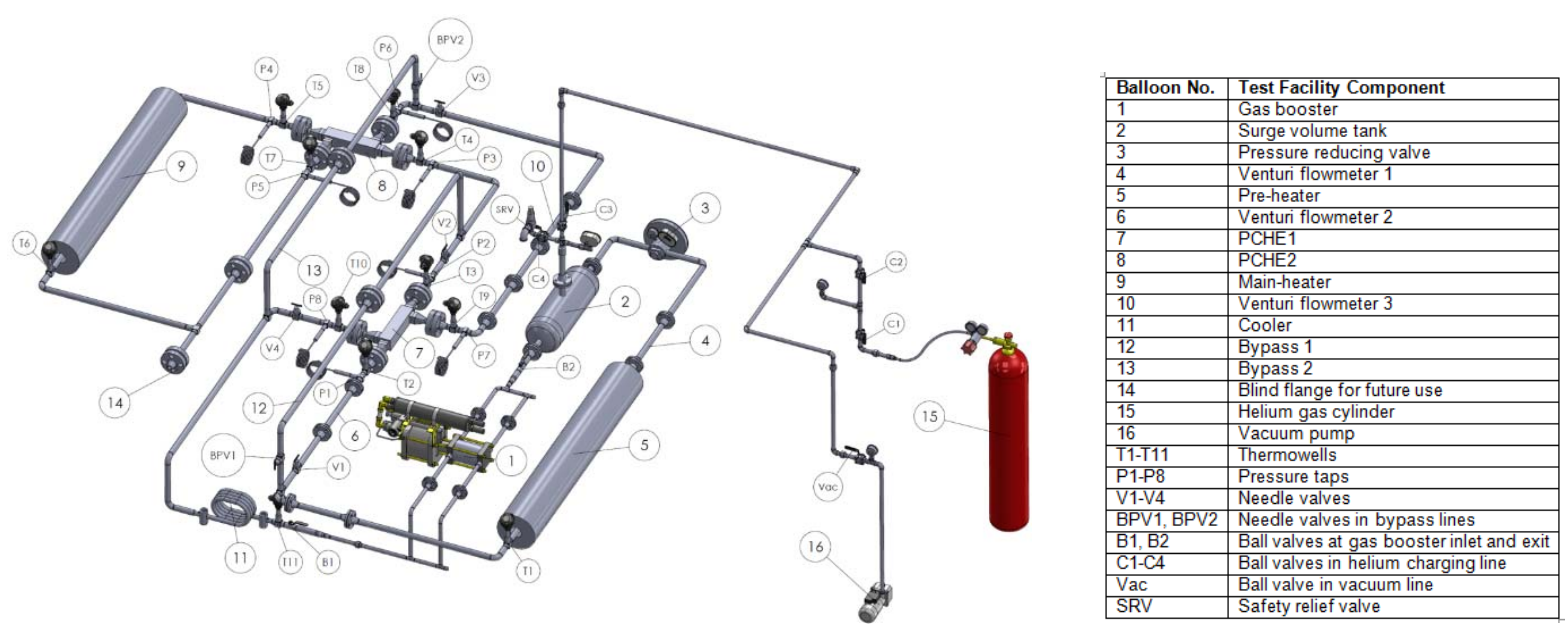

Fig. 3. Layout of the high-temperature helium test facility [5].

\section{Heat Transfer Characteristic of the Test PCHE}

\subsection{Experimental Procedures and Test Conditions}

Thermal-hydraulic performance testing of the straight-channel PCHE was carried out. The experimental testing conditions were the same as the experiments conducted in Ref. [11]. The test loop was initially vacuumed to the desired vacuum pressure of -14 psig using a vacuum pump followed by charging the test loop with helium (a purity rating of 99.9999\%) to a desired pressure from a helium gas cylinder. The 
cooler was turned on during all tests to avoid the high-temperature helium flowing through the gas booster and damaging it. The primary measurements taken on the test PCHE consisted of the inlet and outlet temperatures, pressure drops across both the hot and cold sides, helium mass flow rates on both sides, and gauge pressures at the inlet sections of both sides. Due to the large thermal inertia of the entire test facility, it usually took more than five hours for the test facility to reach the first steady-state condition starting from a room temperature. The criteria of a steady-state condition were based on the standard deviations of the temperature, pressure, and helium mass flow rate measurements. The PCHE operation condition was considered as a steady state when the standard deviations of the four heat exchanger end temperatures were less than $0.5^{\circ} \mathrm{C}$, the static pressure and differential pressure less than $3 \%$, and the helium mass flow rates less than $2 \%$ within 2 minutes at a sampling rate of $8 \mathrm{~Hz}$ [5].

Figure 4 shows a total number of 91 experimental data points in terms of the helium mass flow rates, system pressures, and PCHE hot-side inlet temperatures. The helium mass flow rates were varied from 22 to $39 \mathrm{~kg} / \mathrm{h}$ and the system pressures were raised to $2.7 \mathrm{MPa}$ while the hot-side inlet temperatures were increased from 199 to $450^{\circ} \mathrm{C}$. The heat exchanger thermal duty at each steady state was calculated based on the average thermal duty on both the hot and cold sides, as shown in Fig. 5(a). The maximum heat exchanger thermal duty reached $8.5 \mathrm{~kW}$ among these test conditions. The heat loss from the heat exchanger surfaces is defined as the thermal duty difference between the hot side and cold side of the PCHE. It can be observed from Fig. 5(b) that the ratios of heat loss to the heat exchanger thermal duty were generally less than $15 \%$ except for about 10 runs. 


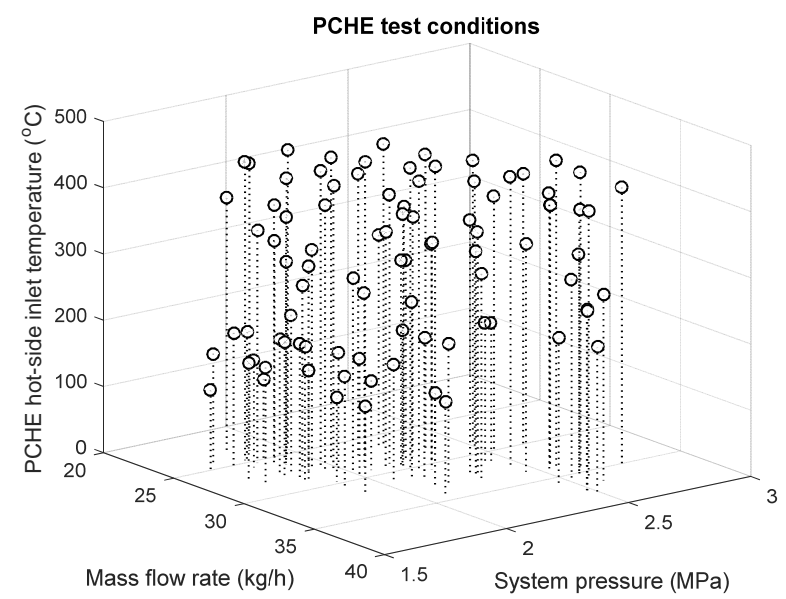

Fig. 4. PCHE test condition.

(a)

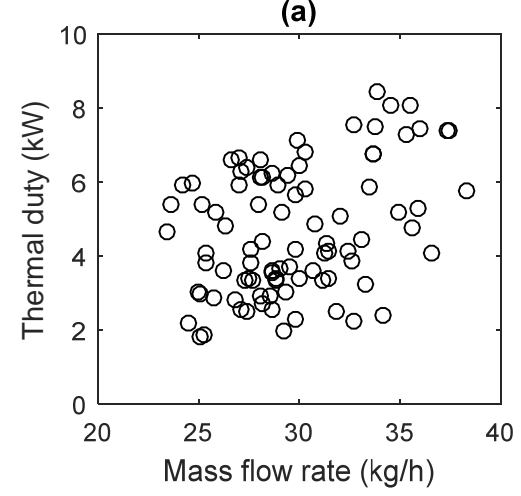

(b)

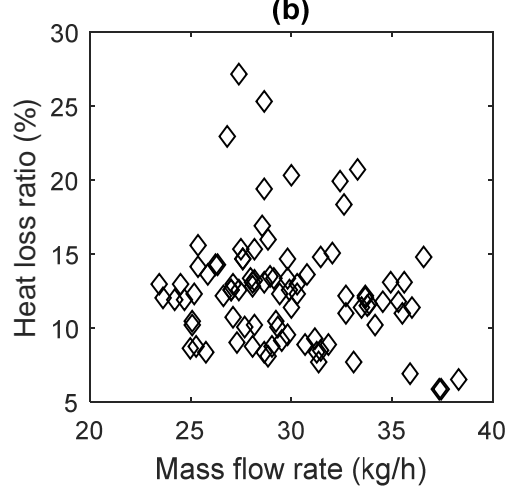

Fig. 5. Heat exchanger thermal duty and heat loss ratio.

\subsection{Experimental Data Reduction Methods}

As mentioned, no local internal fluid and wall temperature measurements were conducted in the present study. Therefore, the local and average convective heat transfer coefficients on both the hot and cold sides of the heat exchanger cannot be obtained. However, with certain assumptions, the equation for evaluating the average heat transfer coefficients of the test heat exchanger can be estimated from the experimental data, including two inlet temperatures, two outlet temperatures, and two helium mass flow rates. Two 
typical methods, namely, a direct method and an indirect method, used to develop formulas for calculating the overall heat transfer coefficient of the PCHE are discussed and compared in this section.

\subsubsection{Direct method}

To obtain the heat transfer coefficients of the test PCHE directly from the experimental data, three major assumptions were made: 1 . Temperature distributions on both the hot and cold sides and solid plates were linear along the heat exchanger flow length; 2. Heat transfer coefficients on both the hot and cold sides were the same for each steady-state condition (i.e., thermal resistances due to convection were approximately the same on both the hot and cold sides); 3 . The effect of the flow and thermal entrance region was neglected; and 4 . The thermal resistance due to the heat conduction in the solid plates was neglected. The heat transfer rate across the PCHE is given by:

$$
\dot{Q}=U A_{s} \Delta T_{l m}
$$

where $\dot{Q}, U, A_{s}$, and $\Delta T_{l m}$ denote the heat transfer rate, overall heat transfer coefficient, heat transfer area, and log-mean temperature difference (LMTD), respectively. The overall heat transfer coefficient is a function of the flow geometry, fluid properties, and solid plate material, which can be obtained from the experimental data using Eq. (1). From the PCHE fabrication aspects, the hot-side heat transfer area, $A_{s, h}$, and the cold-side heat transfer area, $A_{s, c}$, are essentially the same (i.e., $A_{s, h}=A_{s, c}=A_{s}$ ). The overall thermal resistance can be expressed as:

$$
\frac{1}{U A_{s}}=\frac{1}{h_{h} A_{s, h}}+\frac{1}{h_{c} A_{s, c}}+R_{w} .
$$


Applying the aforementioned assumptions, the plate thermal resistance term $R_{w}$ was neglected and the average convective heat transfer coefficient on either side can be estimated from experimental data as:

$$
h_{h}=h_{c}=2 U
$$

\subsubsection{Indirect method}

An indirect method used for determining the average heat transfer coefficient in the PCHE is described. For a determined fluid (i.e., helium), the Nusselt number is a function of the Reynolds number only. The Prandtl number of helium was treated as a constant since its variations over the testing temperature range in the current study were less than $1 \%$. The heat transfer coefficient for either side can be calculated from the Nusselt number that can be expressed as:

$$
\mathrm{Nu}=c \operatorname{Re}^{a}
$$

where $a$ and $c$ are constants. Therefore, the average heat transfer coefficient $h$ is given by:

$$
h=\frac{\mathrm{Nu} \lambda}{d_{h}}=\frac{c \operatorname{Re}^{a} \lambda}{d_{h}},
$$

where $\lambda$ is the thermal conductivity of the fluid and $d_{h}$ is the channel hydraulic diameter. Substituting Eq. (5) into Eq. (2) yields:

$$
\frac{1}{U d_{h}}=\frac{1}{c \operatorname{Re}_{h}^{a} \lambda_{h}}+\frac{1}{c \operatorname{Re}_{c}^{a} \lambda_{c}}+\frac{R_{w} A_{s}}{d_{h}}
$$


where $U, \operatorname{Re}, R_{w}$, and $\lambda$ can be obtained directly from the experimental data; $A_{s}$ and $d_{h}$ are geometrical parameters. Two unknown constants $a$ and $c$ can be solved using a nonlinear regression method to minimize the residual $S$ as:

$$
S=\sum_{j=1}^{N}\left[\frac{1}{U_{j} d_{h}}-\frac{R_{w, j} A_{s}}{d_{h}}-\frac{1}{c \operatorname{Re}_{h, j}^{a} \lambda_{h, j}}-\frac{1}{c \operatorname{Re}_{c, j}^{a} \lambda_{c, j}}\right]^{2},
$$

where $N$ is the total number of the available experimental data points. Once $a$ and $c$ are determined, the Nusselt number correlation (i.e., Eq. (4)) is obtained. The heat transfer coefficients for both the hot and cold sides can be calculated separately from Eq. (4).

\subsection{Experimental Data Analysis and Discussions}

The Nusselt numbers for both the hot and cold sides using the direct method are plotted in Fig. 6 with uncertainty bars representing the propagated errors. The largest uncertainty among all the data points was 13\%. It was observed that the Nusselt numbers on the hot and cold sides follows the same trend well. There were some differences between the hot side and cold side, which can be attributed to the differences in the thermal conductivity of helium on both sides since the heat transfer coefficients were assumed the same for both sides. 


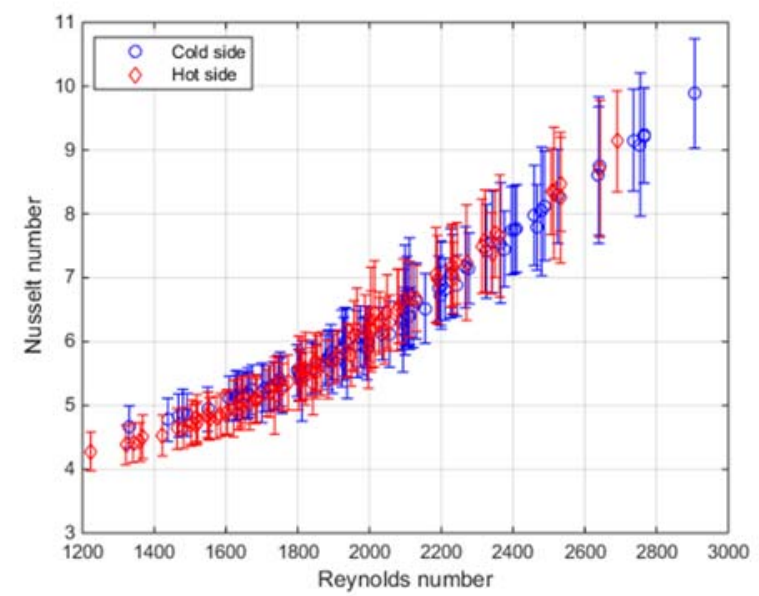

Fig. 6. Nusselt numbers on both the hot and cold sides using the direct method.

It can be seen from Fig. 6 that the Reynolds number were varied from 1,200 to 2,900, covering the laminar flow and laminar-to-turbulence flow transition regimes. The trend of the Nusselt number indicated that a change in the Reynold number between 1,800 and 2,000. To find the best fit correlations for the Nusselt numbers, two correlations were fit for the two regimes. Residuals of the correlations were calculated based on the root-sum-squared formula. Figure 7 shows the residuals plotted against the critical Reynolds numbers that presumably separate the two regimes. It was evident that the minimum residual occurred at the Reynolds number of about 1,850. Therefore, it appears reasonable to assume that the critical Reynold number was approximately 1,850. For the PCHE used in the helium-helium conditions, the Nusselt number is essentially a power function of the Reynolds number only. The best fit correlations for the total 182 data points on both the hot and cold sides with the critical Reynold number of 1,850 were proposed as:

$$
\mathrm{Nu}= \begin{cases}(0.01352 \pm 0.0094) \operatorname{Re}^{(0.80058 \pm 0.0921)} & 1,200 \leq \mathrm{Re} \leq 1,850 \\ \left(3.6361 \times 10^{-4} \pm 7.855 \times 10^{-5}\right) \operatorname{Re}^{(1.2804 \pm 0.0273)} & 1,850<\operatorname{Re} \leq 2,900\end{cases}
$$




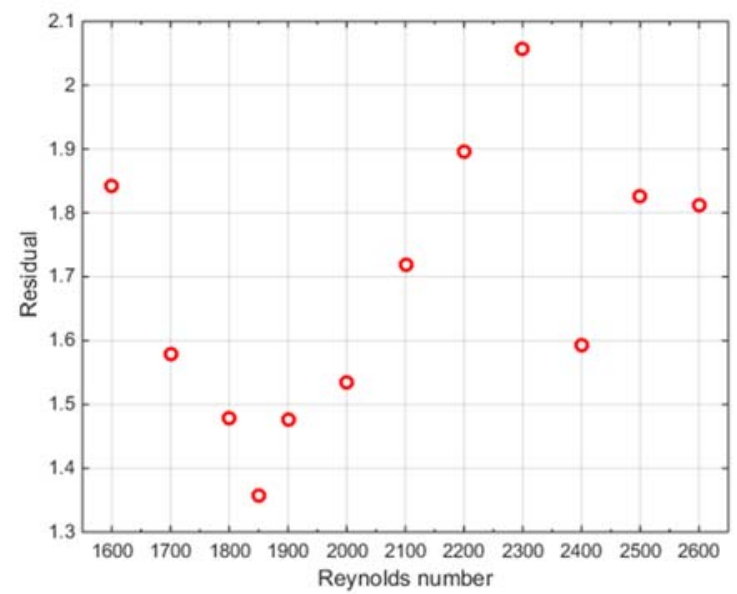

Fig. 7. Residuals of fitted functions with different onset points.

Next, for the indirect method, validation for a fit correlation was considered in the data reduction process. The correlation was obtained via a nonlinear regression method using 76 data sets randomly selected from the total 91 data sets. The other 15 data sets were reserved for validating the fit correlation. Following the heat transfer data reduction method illustrated in Section 3.2.2, the fit correlation was obtained as:

$$
\mathrm{Nu}=\left\{\begin{array}{ll}
(0.047516 \pm 0.015662) \operatorname{Re}^{(0.633151 \pm 0.044606)} & 1,200 \leq \operatorname{Re} \leq 1,850 \\
\left(3.680123 \times 10^{-4} \pm 1.184389 \times 10^{-4}\right) \operatorname{Re}^{(1.282182 \pm 0.042068)} & 1,850<\operatorname{Re} \leq 2,900
\end{array} .\right.
$$

Both of the coefficients in Eqs. (8) and (9) included confidence intervals with a confidence level of 95\%. A comparison of the results obtained from the direct and indirect methods is shown in Fig. 8. It can be seen that the Nusselt numbers obtained from the nonlinear regression method were slightly larger than those obtained from the direct method. The largest deviation was $6.5 \%$ occurred at the lowest Reynolds number point. 


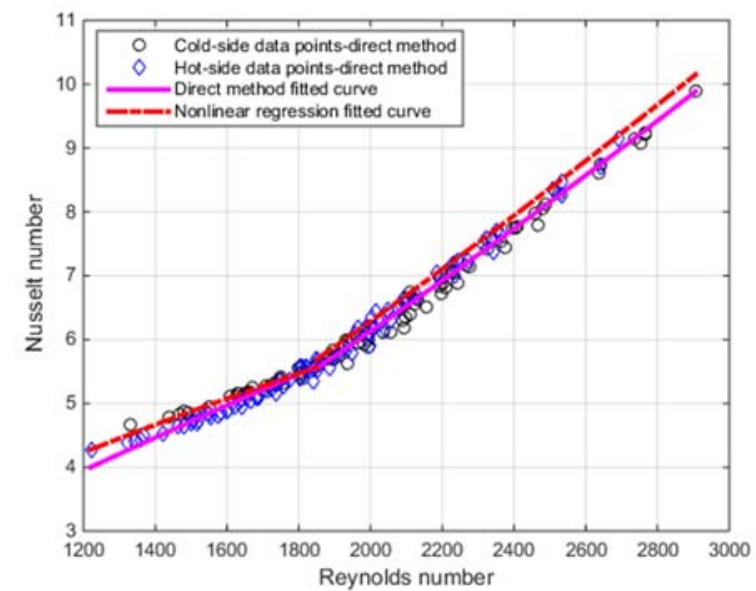

Fig. 8. Comparison of the results obtained from the direct and indirect methods.

A comparison of the overall heat transfer coefficients obtained directly from the 15 reserved sets of the experimental data and the calculated overall heat transfer coefficients using Eqs. (8) and (9) is presented in Fig. 9. The comparison showed that the calculated overall heat transfer coefficients were in good agreement with the experimental overall heat transfer coefficients. The differences between the calculated values and experimental data were within 5\%. Although both the direct and indirect methods can predict the heat transfer coefficients for the tested PCHE well, only the correlation developed using the nonlinear regression method, i.e., Eq. (9), was implemented in the dynamic code for the transient simulations to be discussed in the next section since it is the best fitted correlation from the experimental data. 


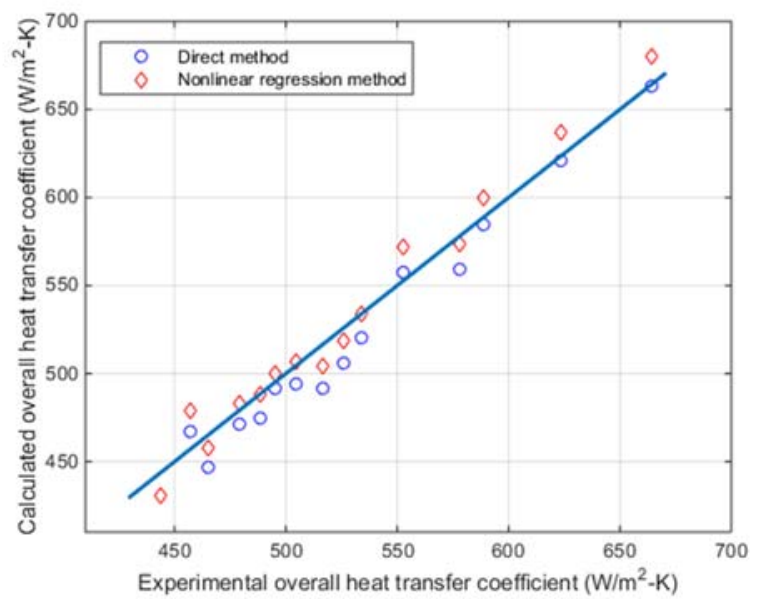

Fig. 9. Comparison of the overall heat transfer coefficients obtained from fitted models and experiments.

\section{Dynamic Response of the Test PCHE}

\subsection{Numerical Simulations}

\subsubsection{Mathematical model}

A dynamic model to evaluate the performance of the PCHE was developed based on the energy balance of a two-stream countercurrent flow heat exchanger with the following assumptions:

1. Uniform helium low distribution into the flow channels;

2. Negligible axial heat conduction in both the solid plates and working fluid;

3. Negligible heat loss to the surroundings via the heat exchanger surfaces;

4. Constant specific heat for helium; and

5. Negligible thermal resistance due to the heat conduction resistance in the solid plates between the hot and cold channels because of the thin plates and large thermal conductivity of the plate material (i.e., Alloy 617). 
Two control volumes for the fluids inside two neighboring flow channels and a control volume in one of the non-etched portion on the solid plate between the two flow channels are illustrated in Fig. 10. Based on the aforementioned assumptions, integrating the energy balance equations over the PCHE, one is able to derive the following equations [10]:

\begin{tabular}{|l|l|l|}
\hline$x_{c}^{T_{c}^{\text {out }}} \cdot \mathrm{d} x$ & $T_{c}^{\text {in }} \quad$ Cold side \\
\hline & & Plate \\
\hline$T_{h}^{\text {in }} \rightarrow$ & $T_{h}^{\text {out }}$ Hot side \\
\hline
\end{tabular}

Fig. 10. Control volumes for two fluid streams and solid plate [10].

For the hot-side fluid:

$$
m_{h} c_{p, h} \frac{\mathrm{d} T_{h}}{\mathrm{~d} t}+\dot{m}_{h} c_{p, h}\left(T_{h}^{\text {out }}-T_{h}^{i n}\right)=(h A)_{h}\left(T_{P}-T_{h}\right)
$$

For the cold-side fluid:

$$
m_{c} c_{p, c} \frac{\mathrm{d} T_{c}}{\mathrm{~d} t}+\dot{m}_{c} c_{p, c}\left(T_{c}^{\text {out }}-T_{c}^{i n}\right)=(h A)_{c}\left(T_{P}-T_{c}\right)
$$

For the solid plate:

$$
m_{P} c_{p, P} \frac{\mathrm{d} T_{P}}{\mathrm{~d} t}=(h A)_{h}\left(T_{h}-T_{P}\right)-(h A)_{c}\left(T_{P}-T_{c}\right)
$$

Here, the subscripts $h, c$, and $P$ denote the PCHE hot-side fluid, cold-side fluid, and solid plate, respectively. $A$ is the total heat transfer area in one specific control volume of the PCHE. Superscripts in and out denote the inlet and outlet temperatures for that control volume, respectively. It is also assumed that the hot and cold channel of interest are aligned on top of each other, and that that the heat transfer path is from the hot channel to the cold channel as shown in Fig. 10. 


\subsubsection{Steady-state simulations}

The numerical model described above was benchmarked in a previous study [10] by comparing the numerical solution with the results obtained from a commercial software DYNSIM. Figure 11 presents the PCHE system nodal structure. A nodalization sensitivity study was performed to determine the effective number of segments, i.e., control volumes for each fluid stream, used in the transient simulations based on one of the experimental steady-state conditions. Five different numbers of segments were used in the nodalization sensitivity study. The helium inlet temperatures on both the hot and cold sides were kept at constants of 465.6 and $207.6^{\circ} \mathrm{C}$, respectively. The temperatures at the hot-side and cold-side outlets were recorded after the system reached a steady state in the calculations using the numerical model. These two outlet temperatures were compared to the values that were obtained from the heat exchanger rating process using the $\varepsilon-\mathrm{NTU}$ method. It can be seen from Table II that these five cases presented similar results without significant deviations. The hot-side and cold-side temperature differences between the values using the $\varepsilon-\mathrm{NTU}$ method and the results obtained from the simulations for the five cases were mainly attributed to the fluid thermophysical properties. The fluid thermophysical properties were evaluated based on the average temperature for each helium stream in the heat exchanger rating process while the temperature-dependent properties were employed in each segment in the numerical simulations. Note that increasing the number of segments would considerably increase the computational time. It is observed from Table II that the outlet temperature differences on both sides tended to diminish when the total number of segments were greater than 500 . Therefore, a total of 500 segments were selected for the PCHE to simulate the transient scenarios in this study. 


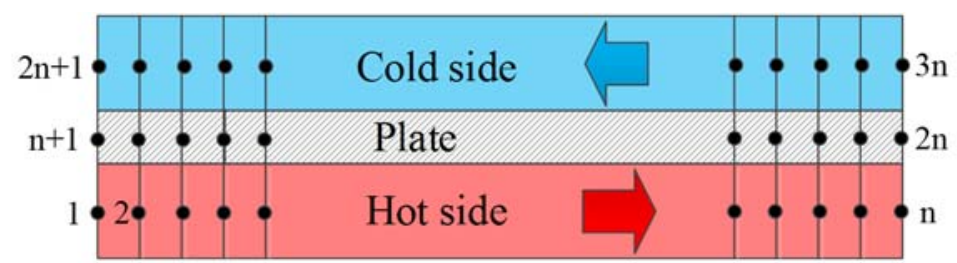

Fig. 11. PCHE nodal structure.

Table II. Results of nodalization sensitivity study

\begin{tabular}{|l|c|c|c|c|c|c|}
\hline & \multirow{2}{*}{\begin{tabular}{c} 
PCHE \\
$\varepsilon-\mathrm{NTU}$ \\
\cline { 5 - 8 }
\end{tabular}} & \multicolumn{5}{|c|}{ Number of segments } \\
\cline { 5 - 8 } & rating value & 50 & 100 & 300 & 500 & 1000 \\
\hline Hot-side outlet temperature $\left({ }^{\circ} \mathrm{C}\right)$ & 294 & 294 & 293.7 & 293.6 & 293.6 & 293.5 \\
\hline Cold-side outlet temperature $\left({ }^{\circ} \mathrm{C}\right)$ & 379.2 & 379.5 & 379.2 & 379.0 & 379.0 & 379.0 \\
\hline
\end{tabular}

To predict the dynamic behavior of the straight-channel PCHE, steady-state operating parameters were first calculated by assuming constant helium fluid properties. The input parameters for the calculation included the helium inlet temperatures on both the hot and cold sides. The temperature distributions inside the PCHE, as shown in Fig. 12, were obtained by dividing one hot helium stream, one cold helium stream, and one plate into 500 axial segments along the helium flow directions. The steady-state temperatures inside the PCHE and helium mass flow rates can serve as the initial condition for the transient simulations. 


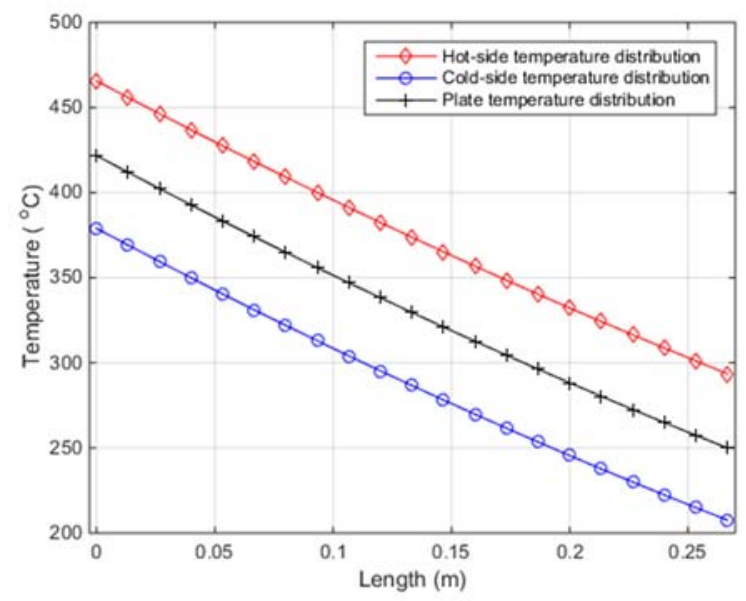

Fig. 12. Temperature distributions inside the reduced-scale straight-channel PCHE.

\subsubsection{Transient simulations}

A series of transient scenarios were performed to study the dynamic behavior of the PCHE and also to assess the applicability of the dynamic model for use in the straight-channel PCHE under hightemperature helium-helium conditions. For helium inlet temperature variations, the combinations of step change on one side and linear ramp change on the other side were performed. Similarly, the combinations of step change in helium mass flow rate on one side and linear ramp change on the other side were carried out. For the PCHE subject to combinations of the helium inlet temperature and mass flow rate variations, four cases in which the PCHE would experience the highest and lowest temperatures among the variation combinations were conducted. The detailed numerical simulation matrix is listed in Table III. 
Table III. Numerical simulation matrix

\begin{tabular}{|c|c|c|}
\hline Case & Hot-side inlet & Cold-side inlet \\
\hline & \multicolumn{2}{|c|}{ Temperature variations } \\
\hline HSICRD & $50^{\circ} \mathrm{C}$ step increase & $50^{\circ} \mathrm{C}$ linear ramp decrease within 10 seconds \\
\hline HSDCRI & $50^{\circ} \mathrm{C}$ step decrease & $50^{\circ} \mathrm{C}$ linear ramp increase within 10 seconds \\
\hline HRICSD & $50^{\circ} \mathrm{C}$ linear ramp increase within 10 seconds & $50^{\circ} \mathrm{C}$ step decrease \\
\hline \multirow[t]{2}{*}{ HRDCSI } & $50^{\circ} \mathrm{C}$ linear ramp decrease within 10 seconds & $50^{\circ} \mathrm{C}$ step increase \\
\hline & \multicolumn{2}{|c|}{ Helium mass flow rate variations } \\
\hline HSICRI & $20 \%$ step increase & $20 \%$ linear ramp increase within 10 seconds \\
\hline HSDCRD & $20 \%$ step decrease & $20 \%$ linear ramp decrease within 10 seconds \\
\hline HRICSI & $20 \%$ linear ramp increase within 10 seconds & $20 \%$ step increase \\
\hline \multirow[t]{2}{*}{ HRDCSD } & $20 \%$ linear ramp decrease within 10 seconds & $20 \%$ step decrease \\
\hline & \multicolumn{2}{|c|}{ Inlet temperature combined with helium mass flow rate variations } \\
\hline \multirow{2}{*}{ TSI-HSICSD } & $50^{\circ} \mathrm{C}$ step increase & $50^{\circ} \mathrm{C}$ step increase \\
\hline & $20 \%$ flow rate step increase & $20 \%$ flow rate step decrease \\
\hline \multirow{2}{*}{ TRI-HRICRD } & $50^{\circ} \mathrm{C}$ linear ramp increase within 10 seconds & $50^{\circ} \mathrm{C}$ linear ramp increase within 10 seconds \\
\hline & $20 \%$ flow rate linear ramp increase within 10 seconds & $20 \%$ flow rate linear ramp decrease within 10 seconds \\
\hline \multirow{2}{*}{ TSD-HSDCSI } & $50^{\circ} \mathrm{C}$ step decrease & $50^{\circ} \mathrm{C}$ step decrease \\
\hline & $20 \%$ flow rate step decrease & $20 \%$ flow ratestep increase \\
\hline \multirow{2}{*}{ TRD-HRDCRI } & $50^{\circ} \mathrm{C}$ linear ramp decrease within 10 seconds & $50^{\circ} \mathrm{C}$ linear ramp decrease within 10 seconds \\
\hline & $20 \%$ flow rate linear ramp decrease within 10 seconds & $20 \%$ flow rate linear ramp increase within 10 seconds \\
\hline
\end{tabular}

a. Inlet temperature variations

The dynamic behavior of the PCHE subject to the helium inlet temperature variations was first studied.

The helium inlet velocities were kept at 26.6 and $23.2 \mathrm{~m} / \mathrm{s}$ on the hot side and cold side, respectively.

Figures 13 shows the hot-side and cold-side outlet temperature dynamic response due to four cases of

helium inlet temperature variation combinations initiated at 10 seconds. Note in this figure the solid and dotted lines represent the hot-side and cod-side outlet temperatures, respectively. For both the HSICRD and HRDCSI cases, the cold-side and hot-side helium outlet temperatures had an overshoot at the beginning of the transients. Conversely, undershoots were observed at the outlets on both sides for both the HRICSD and HSDCRI cases. This can be explained that the step changes dominated the transient scenarios during the initial stage. It took approximately 15 seconds for the temperatures to stabilize on both the hot and cold sides for all these temperature variation cases. It also can be seen from Fig. 13 that the outlet temperatures for both the HSICRD and HRICSD cases were the same after the final steady states were reached in the calculations. Similar behavior was presented for the other two temperature 
variation transients. These results indicate that the final steady state is only determined by the degree of the temperature change at the beginning of the transient and is insusceptible to how the temperature change is initiated.

For the HSICRD and HRICSD cases, the hot-side helium outlet temperatures decreased from 293.6 to $271.2^{\circ} \mathrm{C}$ and the helium cold-side outlet temperatures increased from 379 to $387^{\circ} \mathrm{C}$ once new steady states were reached. The effectiveness of the reduced-scale PCHE under the initial steady state was 67\%. The heat exchanger effectiveness was reduced to $64 \%$ after the temperature variation transients. However, the hot-side helium inlet temperature increase combined with the cold-side helium temperature decrease resulted in 38\% more thermal energy transferring from the hot side to the cold side. The deviation of the energy transfer rate was about $6.14 \%$ between the hot side and the cold side in the final steady-state condition. For the HSDCRI and HRDCSI cases, the hot-side helium outlet temperature increased to $313^{\circ} \mathrm{C}$ and the cold-side helium outlet temperature decreased to $365.8^{\circ} \mathrm{C}$. The combination of the hot-side temperature decrease and the cold-side temperature increase reduced $38.6 \%$ of the thermal energy transferring from the hot side to the cold side. The hot-side and cold-side energy transfer rate was within a deviation of 5.5\%. Compared to the initial operation parameters, the heat exchanger effectiveness slightly was increased to $68 \%$.

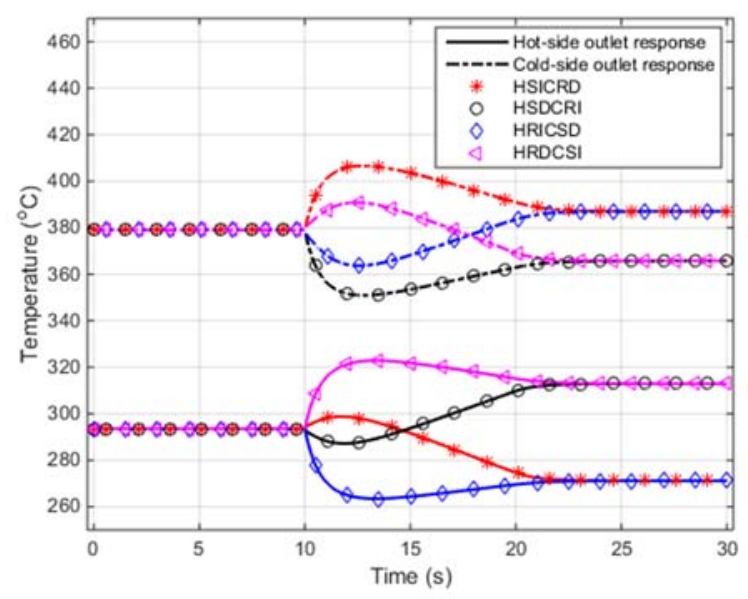

Fig. 13. PCHE dynamic response to the inlet temperature variations. 


\section{b. Helium mass flow rate variations}

In this section, dynamic response of the simulated PCHE subject to four cases of helium mass flow rate variation combinations was analyzed. The four helium mass flow rate transient scenarios are listed in Table III. Only changes in the helium mass flow rate were considered for each instance. That is, the helium inlet temperatures were kept constant on both sides for these simulations. Figure 14 shows the dynamic temperature response when the helium mass flow rates on both sides were changed at 10 seconds into the simulations. It can be seen that the helium outlet temperatures on both sides increased in the cases where the helium mass flow rates had a step increase on the hot side or a step decrease on the cold side at the beginning of the transients. The same final steady-state conditions were reached for either the helium mass flow rate increase or decrease on both sides in the calculations. The hot-side helium outlet temperatures decreased from 293.6 to $290.9^{\circ} \mathrm{C}$ and the cold-side outlet helium temperatures increased from 379 to $382.2^{\circ} \mathrm{C}$ for the helium mass flow rate increase and decrease combinations, respectively. More thermal energy carried by the hot fluid would go into the heat exchanger due to the increase of the helium mass flow rate on the hot side while more thermal energy would be removed from the heat exchanger due to the increase of the cold-side helium mass flow rate. The hot-side heat transfer coefficients increased by $26.8 \%$ (i.e., from 1,167 to $1,480 \mathrm{~W} /\left(\mathrm{m}^{2}{ }^{\circ} \mathrm{C}\right)$ ) and decreased by $13.2 \%$ (i.e., from 1,167 to $\left.1,013 \mathrm{~W} /\left(\mathrm{m}^{2}{ }^{\circ} \mathrm{C}\right)\right)$ due to the hot-side helium mass flow rate increase and decrease, respectively. The cold-side heat transfer coefficients for the helium mass flow rate step increase and step decrease rose by $26.3 \%$ (i.e., from 1,201 to $1,517 \mathrm{~W} /\left(\mathrm{m}^{2} \cdot{ }^{\circ} \mathrm{C}\right)$ ) and reduced by $18.7 \%$ (i.e., from 1,201 to 976 $\left.\mathrm{W} /\left(\mathrm{m}^{2} \cdot{ }^{\circ} \mathrm{C}\right)\right)$, respectively. The increasing helium mass flow rates on both sides resulted in $1.8 \%$ more thermal energy transferring from the heat exchanger's hot side to the cold side. It also can be calculated that $1.7 \%$ more thermal energy was exchanged for the decreasing helium mass flow rate combinations. The deviations of the energy transfer rate were approximately 0.11 and $0.06 \%$ after the steady states were reached for the helium mass flow rate increase and decrease combinations, respectively. 

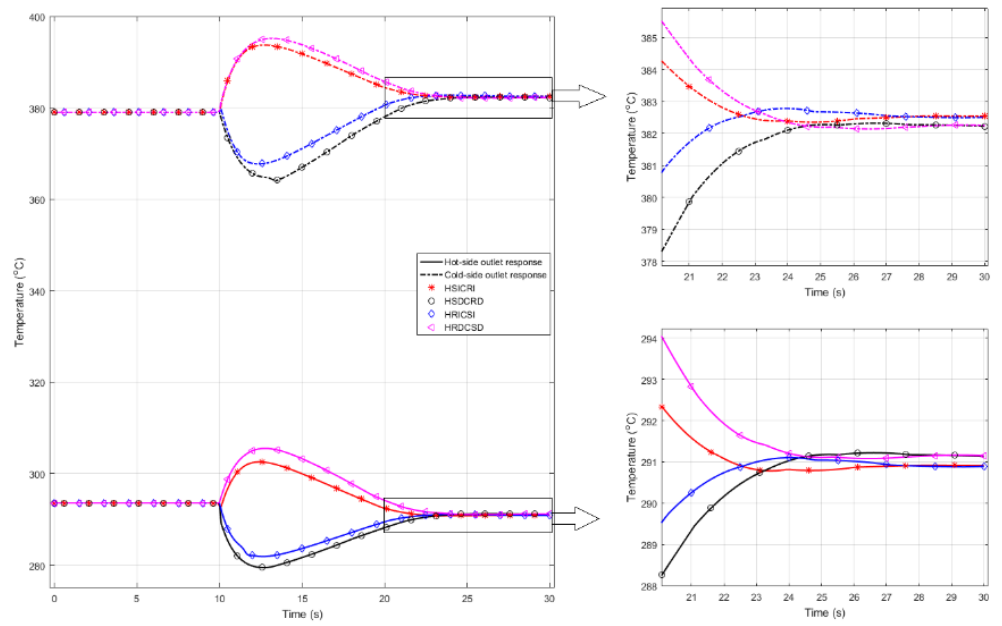

Fig. 14. PCHE dynamic response to the helium mass flow rate variations.

c. Inlet temperature and helium mass flow rate variation combinations

The PCHE may experience a condition of the highest temperature when both of the helium inlets encounter a temperature increase, helium mass flow rate increases on the hot side but decreases on the cold side. On the contrary, the PCHE would undergo a condition of the lowest temperature. Both conditions were studied in this section with the same degrees of change as the helium inlet temperature and mass flow rate variations conducted in the previous sections (i.e., $50^{\circ} \mathrm{C}$ step changes and helium mass flow rate 20\%-step changes). The transient test matrix is listed in Table III. Figure 15 presents the helium outlet temperature evolutions for these four transient scenarios. The highest helium outlet temperatures on the cold and hot sides were 471.4 and $374.9^{\circ} \mathrm{C}$, respectively. The lowest helium outlet temperatures on the cold and hot side were 284.2 and $219.3^{\circ} \mathrm{C}$, respectively. The heat exchanger effectiveness was increased from $67 \%$ to $83 \%$ for the helium inlet temperature increasing cases while decreased to $49 \%$ for the helium inlet temperature decreasing scenarios. Compared to the initial operating condition, the thermal energy transferred from the heat exchanger's hot side to cold side was reduced by $10 \%$ for the lowest temperature cases. This study provides useful information for the PCHE operation since the 
helium inlet temperature variations combined with its mass flow rate variations would potentially lead to an overheating or overcooling condition for the PCHE and therefore the reactor primary system.

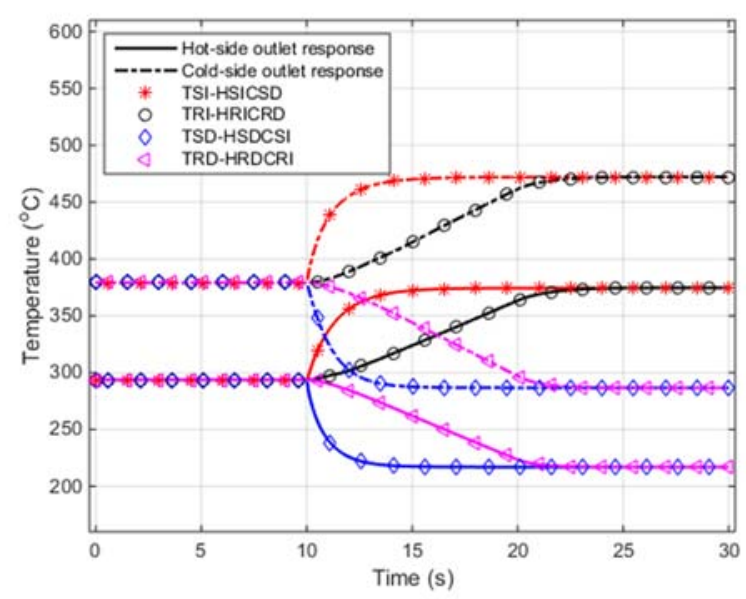

Fig. 15. Temperature evolutions for inlet temperature combined with helium mass flow rate variations.

In all of the transients simulated, for the dynamic response to the $50^{\circ} \mathrm{C}$ variation in the helium inlet temperatures of the test PCHE, the helium inlet temperature increase on the hot side combined with the temperature decrease on the cold side would enhance the heat transfer capability of the heat exchanger while reduce the heat exchanger effectiveness. On the contrary, the helium inlet temperature decrease by $50^{\circ} \mathrm{C}$ on the hot side combined with an inlet temperature increase of $50^{\circ} \mathrm{C}$ on the cold side would increase the heat exchanger effectiveness but reduce the heat transfer capability. For the $20 \%$ of the helium mass flow rate variation combinations, both the heat exchanger effectiveness and heat transfer capability would increase. This dynamic study provides meaningful insight to improve the heat exchanger effectiveness and heat transfer capability when operating it under dynamic conditions. It also provides useful information for developing control strategies to effectively operate the PCHE as an IHX in VHTRs.

\subsection{Experimental Investigations}


The dynamic model discussed above was the first time introduced for straight-channel PCHE transient analyses. Two sets of transient tests were experimentally carried out to assess the applicability of the dynamic model, which are the test PCHE subject to inlet helium temperature increase and decrease on the hot side. A temperature step change on the heat exchanger's inlets is not applicable in the current study due to the limited heater capability and the configuration of the HTHF. For experiments carried out in the HTHF, helium mass flow rates were controlled by a gas booster and helium temperatures by the two electric heaters. Although a temperature step increase or step decrease was not readily achievable, the helium temperatures after exiting the two electric heaters can be adjusted by changing the setting points of the temperature controllers for the heaters and these temperature variations could be captured by the data acquisition system. The hot-side helium inlet temperature was increased in the first test and decreased in the second test. The cold-side helium inlet temperature was maintained at a constant value for both tests. After the PCHE reached a steady state in both tests, temperature variations at the inlet of the hot side were introduced by adjusting the main-heater power via the temperature controller in the HTHF to initiate the transients.

For the transient test initiated by the hot-side helium inlet temperature increase, the cold-side helium inlet temperature and the helium mass flow rates on both sides were kept approximately constants at $49.9^{\circ} \mathrm{C}$ and $31.7 \mathrm{~kg} / \mathrm{h}$, respectively. A polynomial function was used to fit the hot-side helium inlet temperature data, as shown in Fig. 16, and was implemented as the initial condition in the dynamic model. The helium outlet temperatures for both the hot and cold sides obtained from the numerical simulation were compared to the experimental data, as shown in Fig. 17. It was observed that the numerical results predicted the experimental trends well. There were some discrepancies for both the helium outlet temperatures at the initial steady-state condition, which is believed to be primarily attributed to the heat loss from the heat exchanger surfaces. The numerical results on both sides slightly over predicted the experimental values at the initial steady state, i.e., prior to the initiation of the transient around $1000 \mathrm{~s}$. At the end of the transient scenario around 7200 s, the hot-side helium outlet temperatures obtained from the dynamic model and 
experiments were in good agreement. Compared to the experimental results, the computed helium outlet temperatures for the initial steady state and the end of the simulation had deviations of 2.8 and $9.3 \%$, respectively. The heat loss ratio increased from 8.4 to $11.25 \%$ as the hot-side helium inlet temperature increased.

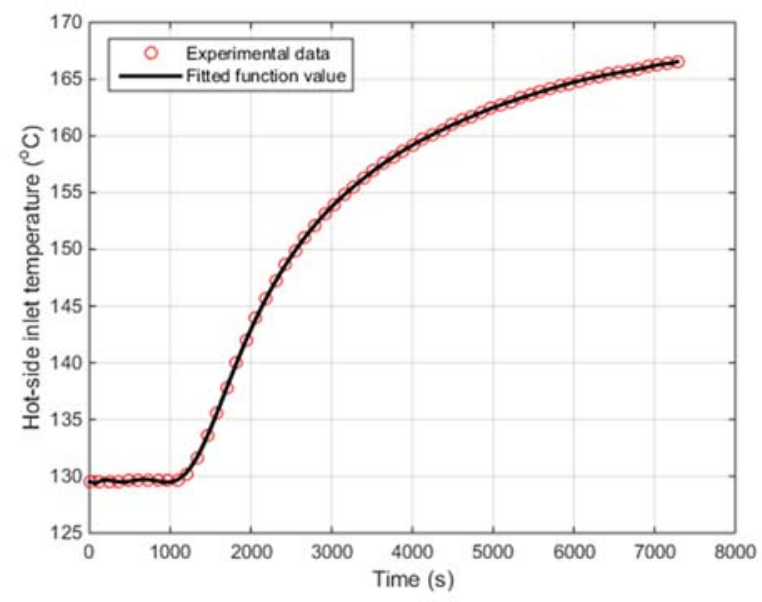

Fig. 16. Curve fitting to the hot-side helium inlet temperature experimental data as a function of time in the first transient test.

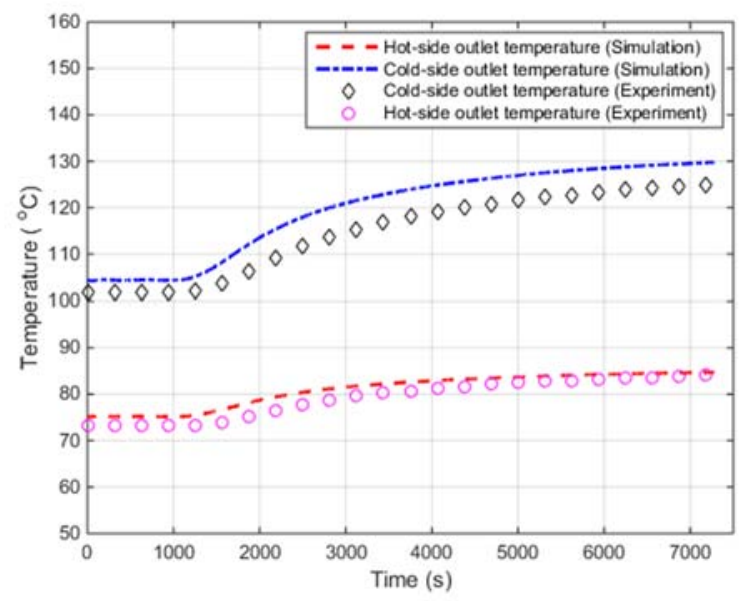

Fig. 17. Helium outlet temperature profiles following the hot-side helium inlet temperature increase shown in Fig. 16. 
A similar analysis methodology was applied to the second transient test, in which the hot-side helium inlet temperature was decreased around 1000 s into the test, as shown in Fig. 18, where the experimental data were curve fitted. The helium outlet temperatures for both the hot and cold sides from the numerical simulation were compared to the experimental data, as shown in. It was observed that the numerical results also predicted the experimental trends well. However, there were even larger discrepancies as compared to the first test, which was also mainly attributed to the heat loss from the heat exchanger surfaces. Compared to the experimental results, the calculated helium outlet temperature differences for the initial steady state and the end of the simulation had deviations of 5.1 and 3.1\%, respectively. The heat loss ratio decreased from 13.0 to $12.3 \%$ as hot-side helium inlet temperature decreased.

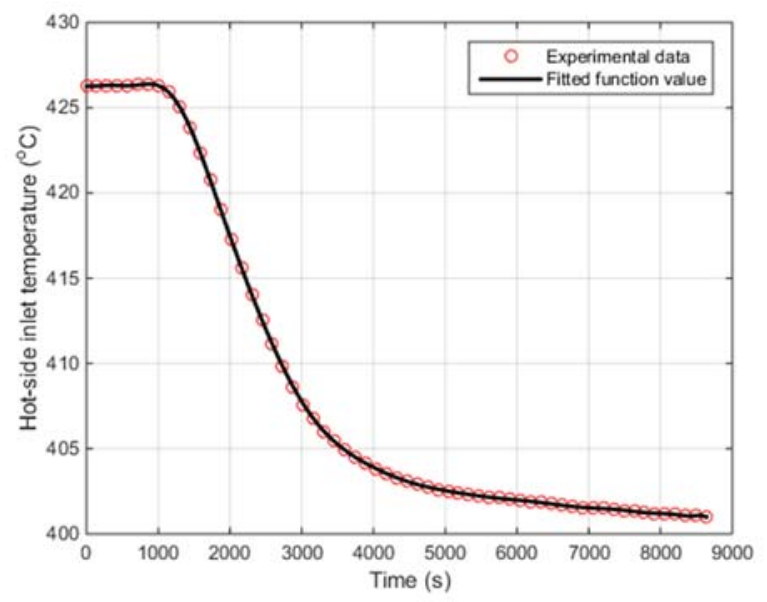

Fig. 18. Curve fitting to the hot-side helium inlet temperature experimental data as a function of time in the second transient test. 


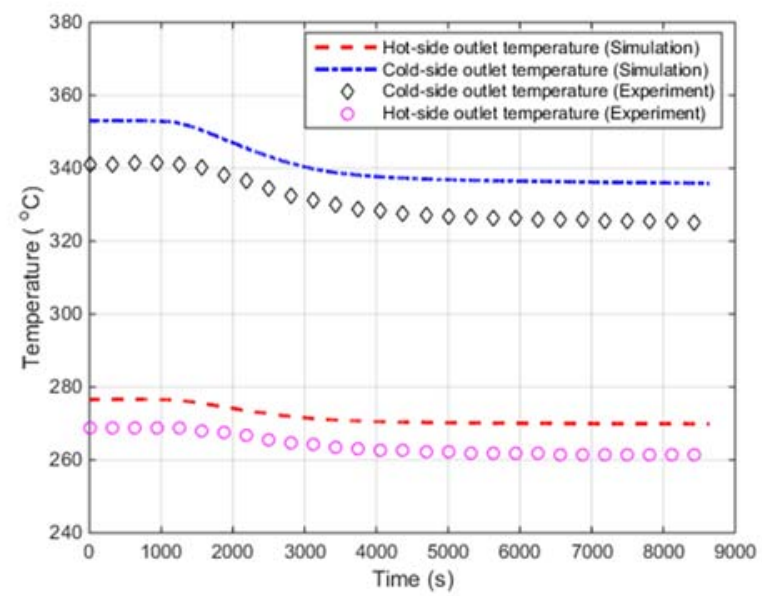

Fig. 19. Helium outlet temperature profiles following the hot-side helium inlet temperature decrease shown in Fig. 18.

\section{Conclusion}

In the current study, a dynamic model benchmarked in a previous study with a commercial software was used to predict the steady-state and transient behaviors of the reduced-scale straight-channel PCHE. Steady-state experimental testing of the PCHE was conducted to obtain the heat transfer correlations of the test PCHE using a direct method and an indirect method. Both heat transfer correlations were able to predict well the overall heat transfer coefficients in the test PCHE. Detailed dynamic response of the straight-channel PCHE, subject to the helium inlet temperature variations and helium mass flow rate variations on both the hot and cold sides, was simulated and analyzed using the dynamic model and the simulation results appear reasonable. In addition, two sets of transient tests were experimentally carried out to examine the dynamic performance of the PCHE and to assess the applicability of the dynamic model. Comparisons of the numerical solutions and experimental results clearly indicate that the dynamic model does a reasonable job in predicting the experimental transient scenarios. The differences in the helium outlet temperatures between the numerical solutions and experimental data are believed primarily due to the heat loss from the heat exchanger, which is not accounted for in the current model. In addition, 
both the numerical and experimental studies provide insights into the PCHE performance and could potentially help devise control strategies for the IHX in VHTRs.

\section{Nomenclature}

a constant in Nusselt number correlation in Eq. (4)

$A_{s} \quad$ surface area

$c \quad$ constant in Nusselt number correlation in Eq. (4)

$c_{p} \quad$ specific heat capacity

$d_{h} \quad$ channel hydraulic diameter

$h \quad$ heat transfer coefficient

$m \quad$ mass in a control volume

$\dot{m} \quad$ helium mass flow rate

$N \quad$ number of experimental data points

$\mathrm{Nu} \quad$ Nusselt number

$\dot{Q} \quad$ thermal energy rate

$R_{w} \quad$ plate thermal resistance

Re Reynolds number

$S \quad$ residual

$t \quad$ time

T temperature

$\Delta T_{l m} \quad$ log-mean temperature difference

$U \quad$ overall heat transfer coefficient

$x \quad$ coordinate in the flow direction, axial direction 
Greek symbols
$\varepsilon \quad$ heat exchanger effectiveness
$\lambda \quad$ helium thermal conductivity

Subscripts

c cold side

$h \quad$ hot side

in inlet of an unit cell

$j \quad j^{\text {th }}$ experiment, index

out outlet of an unit cell

$P \quad$ PCHE solid plate

\section{Acknowledgements}

This research is being performed using funding received from the U.S. Department of Energy Office of Nuclear Energy's Nuclear Energy University Programs. The authors wish to thank Dr. Sai Mylavarapu for his work in the construction of the high-temperature helium test facility and the fabrication of the straightchannel PCHEs.

\section{References}

1. N. Bartel, M. Chen, V.P. Utgikar, X. Sun, I.H. Kim, R. Christensen, and P. Sabharwall, “Comparative Analysis of Compact Heat Exchangers for Application as the Intermediate Heat Exchanger for Advanced Nuclear Reactors,” Annals of Nuclear Energy, 81, pp. 143-149, 2015. 
2. S.J. Bae, J. Lee, Y. Ahn, and J.I. Lee, "Preliminary Studies of Compact Brayton Cycle Performance for Small Modular High-temperature Gas-cooled Reactor System,” Annals of Nuclear Energy, 75, pp. 11-19, 2015.

3. K. Nikitin, Y. Kato, and L. Ngo, "Printed Circuit Heat Exchanger Thermal-hydraulic Performance in Supercritical $\mathrm{CO}_{2}$ Experimental Loop,” International Journal of Refrigeration, 29, pp. 807-814, 2006.

4. P. Sabharwall, E.S. Kim, M. McKellar, N.A. Anderson, and M. Patterson, "Feasibility Study of Secondary Heat Exchanger Concepts for the Advanced High Temperature Reactor,” INL/EXT-1123076, Idaho National Laboratory, 2011.

5. S.K. Mylavarapu, X. Sun, R.E. Glosup, R.N. Christensen, and M.W. Patterson, "Thermal Hydraulic Performance Testing of Printed Circuit Heat Exchangers in a High-temperature Helium Test Facility,” Applied Thermal Engineering, 65, pp. 605-614, 2014.

6. F. Pra, P. Tochon, C. Mauget, J. Fokkens, and S. Willemsen, "Promising Designs of Compact Heat Exchangers for Modular HTRs using the Brayton Cycle,” Nuclear Engineering and Design, 238, pp. 3160-3173, 2008.

7. P. Ravindran, P. Sabharwall, and M.A. Anderson, "Modeling a Printed Circuit Heat Exchanger with RELAP5-3D for the Next Generation Nuclear Plant,” INL/EXT-10-20619, Idaho National Laboratory, 2010.

8. C.C. Lakshmanan and O.E. Potter, “Dynamic Simulation of Plate Heat Exchangers,” International Journal of Heat and Mass Transfer, 33, pp. 995-1002, 1990.

9. F. Sharifi, M.R. Narandji, and K. Mehravaran, “Dynamic Simulation of Plate Heat Exchangers,” International Communications in Heat and Mass Transfer, 22, pp. 213-225, 1995.

10. M. Chen, I.H. Kim, X. Sun, R.N. Christensen, V. Utgikar, and P. Sabharwall, “Transient Analysis of an FHR Coupled to a Helium Brayton Power Cycle,” Progress in Nuclear Energy, 83, pp. 283-293, 2015. 
11. M. Chen, X. Sun, R.N. Christensen, I. Skavdahl, V. Utgikar, and P. Sabharwall, "Pressure Drop and Heat Transfer Characteristics of a High-temperature Printed Circuit Heat Exchanger,” Applied Thermal Engineering, in Revision, 2016.

12. S.K. Mylavarapu, X. Sun, R.N. Christensen, R.R. Unocic, R.E. Glosup, and M.W. Patterson, "Fabrication and Design Aspects of High-temperature Diffusion Bonded Compact Heat Exchangers,” Nuclear Engineering and Design, 249, pp. 49-56, 2012.

13. S.K. Mylavarapu, “Design, Fabrication, Performance Testing, and Modeling of Diffusion Bonded Compact Heat Exchangers in a High-temperature Helium Test Facility,” Ph.D. Thesis, The Ohio State University, 2011. 